\title{
An oxidative amidation and heterocyclization approach for the synthesis of $\beta$-carbolines and dihydroeudistomin $Y$
}

\author{
Suresh Babu Meruva*1,2, Akula Raghunadh¹, Raghavendra Rao Kamaraju \\ U. K. Syam Kumar ${ }^{* 1}$ and P. K. Dubey ${ }^{2}$
}

\section{Full Research Paper}

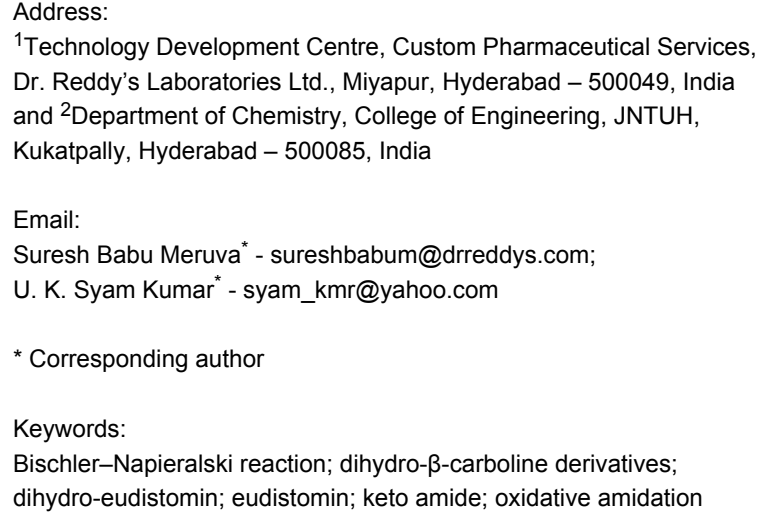

${ }^{1}$ Technology Development Centre, Custom Pharmaceutical Services, Dr. Reddy's Laboratories Ltd., Miyapur, Hyderabad - 500049, India and ${ }^{2}$ Department of Chemistry, College of Engineering, JNTUH, Kukatpally, Hyderabad - 500085, India

Email:

Suresh Babu Meruva* - sureshbabum@drreddys.com;

U. K. Syam Kumar* - syam_kmr@yahoo.com

* Corresponding author

Keywords:

Bischler-Napieralski reaction; dihydro- $\beta$-carboline derivatives;

dihydro-eudistomin; eudistomin; keto amide; oxidative amidation

Beilstein J. Org. Chem. 2014, 10, 471-480.

doi:10.3762/bjoc. 10.45

Received: 13 November 2013

Accepted: 29 January 2014

Published: 25 February 2014

Associate Editor: J. Aubé

(C) 2014 Meruva et al; licensee Beilstein-Institut License and terms: see end of document.

\begin{abstract}
A novel synthetic methodology has been developed for the synthesis of dihydro- $\beta$-carboline derivatives employing oxidative amidation-Bischler-Napieralski reaction conditions using tryptamine and 2,2-dibromo-1-phenylethanone as key starting materials. A number of dihydro- $\beta$-carboline derivatives have been synthesized in moderate to good yields using this methodology. Attempts were made towards the conversion of these dihydro- $\beta$-carbolines to naturally occurring eudistomin alkaloids.
\end{abstract}

\section{Introduction}

$\beta$-Carboline alkaloids [1] are widespread in plants, animals and some are formed naturally in the biological system. Rinehart et al. [2] reported the isolation of $\beta$-carboline alkaloids such as eudistomins [3] and several of its analogues from the active Caribbean colonial tunicate Eudistoma olivaceum. $\beta$-Carboline alkaloids bearing a substituted phenylacetyl group at $\mathrm{C}-1$ position such as eudistomin T (1) [4], eudistomin R (2a) and eudistomin S (2b) [5] were isolated by Cardellina et al. [6], and these compounds exhibit antimicrobial activity. Xestomanzamine A (3) [5] is a $\beta$-carboline alkaloid with an 1-methyl- $1 H$-imidazole- 5-acyl group at the $\mathrm{C}-1$ position and was isolated from the Okinawan marine sponge Xestopongia sp. The other $\beta$-carboline alkaloids reported in the literature are fascaplysin (4) [7], eudistomin A (5a) [3], harmine (5b) [8-10], harmaline (5c) [11], and tetrahydroharmine (5d). Among these, methoxy substituted $\beta$-carboline alkaloids such as $\mathbf{5 b}, \mathbf{5 c}$, and $\mathbf{5 d}$ are indigenously used as hallucinogenic drugs. Some $\beta$-carbolines, notably tryptoline (5e) and pinoline (5f), are produced naturally in the human body. Recently Heonjoong et al. isolated new $\beta$-carboline-based metabolites, designated as eudistomins $\mathrm{Y}_{1}-\mathrm{Y}_{7}$ 
$(\mathbf{6 a}-\mathbf{g})$ [12-14] (Figure 1), from the tunicate of the genus Eudistoma and posses a benzoyl group at the $\mathrm{C}-1$ position of the $\beta$-carboline structural framework. Eudistomins $\mathrm{Y}_{1}-\mathrm{Y}_{7}(\mathbf{6 a}-\mathbf{6 g})$ were evaluated for their antibacterial activity. Eudistomin $\mathrm{Y}_{6}$ (6f) exhibits moderate antibacterial activity against the Grampositive bacteria Staphylococcus epidermis and Bacillus subtilis.

\section{Results and Discussion}

There are several approaches known in literature for the synthesis of $\beta$-carbolines. Most of the syntheses of eudistomin T (1) are generally carried out either from indole or its suitably substituted derivatives. The acylation of 2-(3-indolyl)ethyl isocyanide with phenylacetyl chloride followed by cyclization and aromatization is well documented in the literature for the synthesis of 1-benzoyldihydro- $\beta$-carbolines. The cyclization of the adduct, formed by the reaction of tryptamine with appropriately substituted 1,2,3-tricarbonyl compounds or with glyoxylic acid derivatives under Pictet-Spengler conditions is also reported in literature for the syntheses of eudistomin $\mathrm{T}$ (1) and eudistomin S (2b). Jenkins and co-workers reported the synthesis of fascaplysin (4) by the reaction of tryptamine with phenylacetyl chloride and carried out the aromatization under photo-oxidation conditions $[15,16]$. Lindsley and co-workers [17] reported the synthesis of eudistomins $\mathrm{Y}_{1}-\mathrm{Y}_{7}(\mathbf{6 a}-\mathbf{6 g})$ under microwave conditions [18]. Considering the complexity involved in the synthesis of several of the starting materials used in the preparation of carbolines, especially tricarbonyl compounds, an alternate approach for the synthesis of $3 H-\beta$ carboline is sought after. Herein we described our successful efforts towards the synthesis of 1-benzoyldihydro- $\beta$-carbolines or dihydroeudistomin. The previously developed synthetic methodologies in our laboratory [19-24] were utilized for the synthesis of eudistomin Y (6) and its analogues

The disconnection approach employed in the synthesis of 1-benzoyl- $\beta$-carboline is described in Scheme 1. Accordingly eudistomin Y (6) could be obtained by the aromatization of<smiles>[R]c1cc2[nH]c3c(C(=O)Cc4ccccc4)nccc3c2cc1[R]</smiles>

$1 \mathrm{R}^{1}, \mathrm{R}^{2}=\mathrm{H}$ eudistomin $\mathrm{T}$ 2a $R^{1}=H, R^{2}=B r$ eudistomin $R$ 2b $R^{1}=B r, R^{2}=H$ eudistomin $S$<smiles>Cn1cncc1C(=O)c1nccc2c1[nH]c1ccccc12</smiles>

xestomanzamine A 3<smiles></smiles>

fascaplysin 4

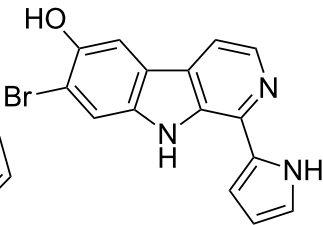

eudistomin A 5a<smiles>COc1ccc2c(c1)[nH]c1c(C)nccc12</smiles>
harmine $\mathbf{5 b}$<smiles>c1ccc2c3c([nH]c2c1)CNCC3</smiles>

tryptoline $5 \mathrm{e}$<smiles>COc1ccc2c3c([nH]c2c1)C(C)=NCC3</smiles>
harmaline $5 \mathrm{c}$

$\mathrm{MeO}$<smiles>Cc1ccc2[nH]c3c(c2c1)CCNC3</smiles><smiles>COc1ccc2c3c([nH]c2c1)C(C)NCC3</smiles>

tetrahydroharmine $\mathbf{5 d}$<smiles>[R]c1cc2[nH]c3c(C(=O)c4cc([R])c(O)c([R4])c4)nccc3c2cc1[R]</smiles>

eudistomin $Y_{1}-Y_{7}(6 \mathbf{a}-6 \mathrm{~g})$

6a: $R^{1}=H \quad R^{2}=H \quad R^{3}=H \quad R^{4}=H$

6b: $R^{1}=B r \quad R^{2}=H \quad R^{3}=H \quad R^{4}=H$

6c: $R^{1}=H \quad R^{2}=H \quad R^{3}=B r R^{4}=H$

6d: $R^{1}=B r \quad R^{2}=H \quad R^{3}=B r R^{4}=H$

6e: $\mathrm{R}^{1}=\mathrm{H} \quad \mathrm{R}^{2}=\mathrm{H} \quad \mathrm{R}^{3}=\mathrm{Br} \mathrm{R}^{4}=\mathrm{Br}$

6f: $R^{1}=B r R^{2}=H \quad R^{3}=B r R^{4}=B r$

6g: $R^{1}=H \quad R^{2}=B r \quad R^{3}=B r \quad R^{4}=B r$

Figure 1: Natural products containing the $\beta$-carboline skeletal.<smiles>[R][X]c1ccc(C(=O)c2nccc3c2[nH]c2ccccc23)cc1</smiles><smiles>[R]c1ccc(C(=O)C2=NCCc3c2[nH]c2ccccc32)cc1</smiles><smiles>CCc1c[nH]c2ccccc12</smiles><smiles>[R]c1ccc(C(=O)C(=O)NCC)cc1</smiles><smiles>NCCc1c[nH]c2ccccc12</smiles><smiles>[R]c1ccc(C(=O)C(Br)Br)cc1</smiles>

11

Scheme 1: Retrosynthetic analysis of 6. 
dihydro- $\beta$-carboline 12. The dihydro- $\beta$-carboline 12 in turn could be synthesized from ketoamide $\mathbf{9}$ under Lewis acid mediated Bischler-Napieralski reaction. The key intermediate, ketoamide 9 required for the synthesis could easily be accessed from tryptamine (10) and appropriately substituted 2,2dibromo-1-phenylethanone (11) $[25,26]$ under oxidative amidation conditions.

The oxidative amidation strategy employed in the current synthesis is previously reported from our group by the reaction of a secondary amine with aryl-2,2-dibromo-1-ethanone under aerial oxidation conditions [24]. Later this methodology was successfully employed in the synthesis of isoquinoline alkaloids [27]. The synthesis of eudistomin Y (6) was initiated with the conversion of the respective 2,2-dibromo-1-phenylethanone to the corresponding Schiff base $\mathbf{1 4}$ by reaction with tryptamine in presence of NaI. The Schiff base on in situ oxidation with cumene hydroperoxide afforded an unstable oxaziridine derivative 15. Ring opening of the oxaziridine derivative $\mathbf{1 5}$ in presence of base afforded ketoiminol 16, which on iminol-amide tautomerism provided the required $\alpha$-ketoamide 9 (Scheme 2).
It was possible to limit the formation of benzamide impurity 17 in the reaction to $<15 \%$ (Table 1 ); however, we were unable to avoid the formation of $\mathbf{1 7}$ under any of the attempted reaction conditions (Scheme 3).

The $\alpha$-ketoamide 9 thus obtained by the oxidative amidation methodology was subjected to a Bischler-Napieralski cyclodehydration reaction in presence of $\mathrm{POCl}_{3}$ [27], however under these conditions 2,9 -dihydro- $\beta$-carboline derivative 7 was obtained as the major product (less than $10 \%$ yield) instead of 4-9-dihydro- $\beta$-carboline derivative 12 (Scheme 4). Our attempts to improve the yield of 7 under Bischler-Napieralski cyclodehydration reaction conditions using $\mathrm{POCl}_{3}$ as the Lewis acid were proved to be futile. This prompted us to test the efficiency of other Lewis acids in this cyclodehydration reaction. The Bischler-Napieralski cyclodehydration reaction was then carried out with different Lewis acids such as $\mathrm{BF}_{3} \cdot \mathrm{Et}_{2} \mathrm{O}, \mathrm{SnCl}_{4}$, $\mathrm{TiCl}_{4}$ etc., in mutiple solvents under various reaction conditions. The best conversion was obtained when the reaction was conducted in $\mathrm{BF}_{3} \cdot \mathrm{Et}_{2} \mathrm{O}$ in ether at $25-30{ }^{\circ} \mathrm{C}$ and the product dihydro- $\beta$-carboline 7 was isolated in $55 \%$ of yield.

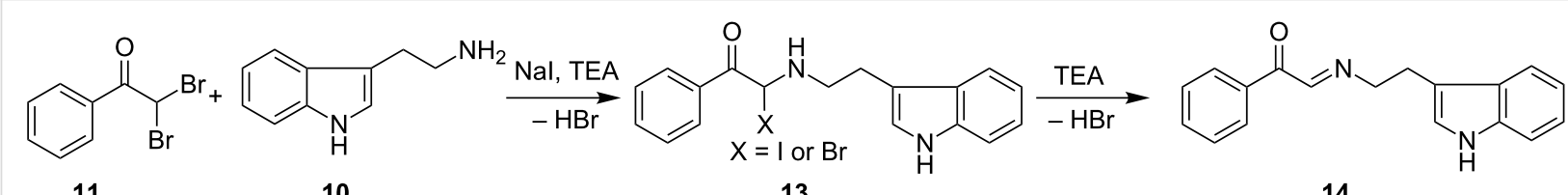

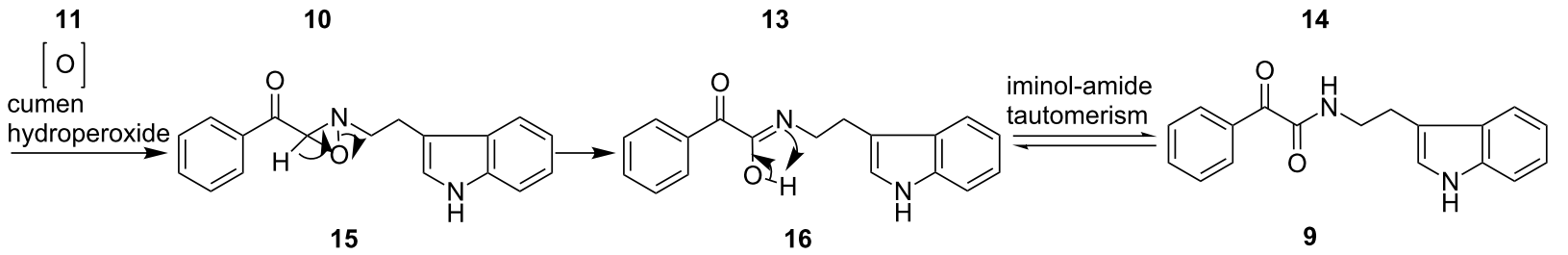

Scheme 2: Plausible mechanism of the oxidative amidation for 9.

Table 1: Effect of solvent and additive on the ratio of 9 and 17

\begin{tabular}{|c|c|c|c|c|c|}
\hline & Base & Solvent /Additives & Reaction time (h) & $\alpha$-Ketoamide $9(\%)$ & Amide $17(\%)$ \\
\hline 1 & TEA & DMSO/Nal & 6 & 60 & 15 \\
\hline 2 & TEA & Sulfolane/Nal & 16 & 52 & 15 \\
\hline 3 & TEA & DMSO & 25 & 42 & 25 \\
\hline
\end{tabular}<smiles>[R]C(=O)C(Br)Br</smiles>

10

\section{DMSO,TEA, Nal,} cumene hydroperoxide, $25-45^{\circ} \mathrm{C}$<smiles>[R]C(=O)C(=O)NCCc1c[nH]c2ccccc12</smiles>

$9 \quad 45-65 \%$<smiles>[R]C(=O)NCCc1c[nH]c2ccccc12</smiles>

$1715 \%$

Scheme 3: Synthesis of $\alpha$-ketoamide 9. 


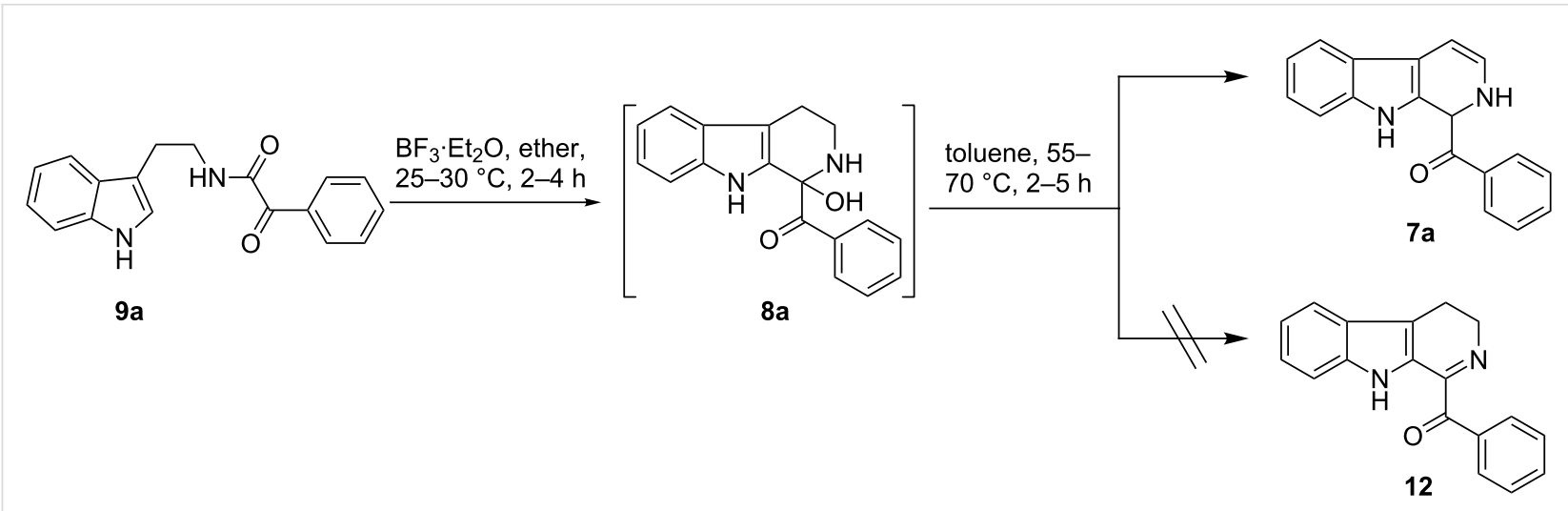

Scheme 4: Synthesis of dihydroeudistomin $Y$ analogues.

The formation of isomeric 2,9-dihydro- $\beta$-carboline derivative 7 as the major product in Bischler-Naperalski reaction conditions is explained in Scheme 5. The initially formed spirocyclic compound 18 undergoes intramolecular rearrangement and afforded the dihydrocarboline framework $\mathbf{1 9}$, which on aromatization yielded 20. Based on the reaction conditions $\mathbf{8}$ can be obtained from 20 after aqueous work-up or at higher temperature heating, 20 undergoes a sequential prototropic migration leading to the formation of 2,9-dihydro- $\beta$-carboline derivative 7 . To the best of our knowledge, this is a novel method for the synthesis of 1-benzoyldihydrocarboline from $\alpha$-ketoamide 9 [28].

The structure of 2,9-dihydro- $\beta$-carboline 7 and 1-hydroxytetrahydro- $\beta$-carboline 8 was confirmed by various NMR techniques such as 2D NMR, COSY as well as HSQC (Figure 2). The coupling of the $\mathrm{H}^{2}$ proton with both $\mathrm{H}^{1}$ and $\mathrm{H}^{3}$ protons is clearly evident in the $\mathrm{COSY}$ for $\mathbf{7 a}$, whereas the $\mathrm{OH}$ proton in 8a shows a high nOe (Scheme 6).<smiles>Cc1[nH]c2ccccc2c1CCNC(=O)C(=O)c1ccccc1</smiles>

$9 a$

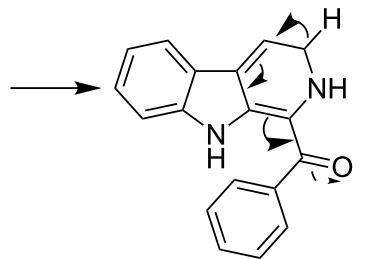

20<smiles>O=C(c1ccccc1)C1(O)NCCC12C(=O)Nc1ccccc12</smiles>

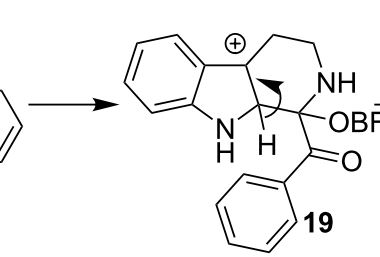

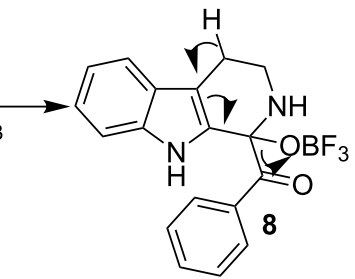<smiles>C=C=C(CC)C(C)(O)c1ccccc1</smiles>

21<smiles>O=C(c1ccccc1)C1NC=Cc2c1[nH]c1ccccc21</smiles>

$7 a$

Scheme 5: Plausible mechanism for the formation of 7.<smiles>O=C(c1ccccc1)C1(O)NCCc2c1[nH]c1ccccc21</smiles>

$8 \mathbf{a}$

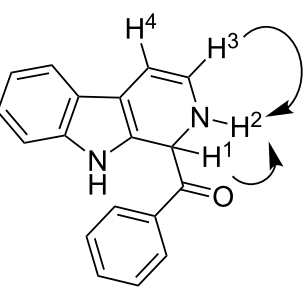

$7 a$<smiles>C#CC=C</smiles>

21

Scheme 6: Rearrangement of $8 \mathbf{a}$ into $7 \mathbf{a}$ and coupling interactions of $7 \mathbf{a}$. 

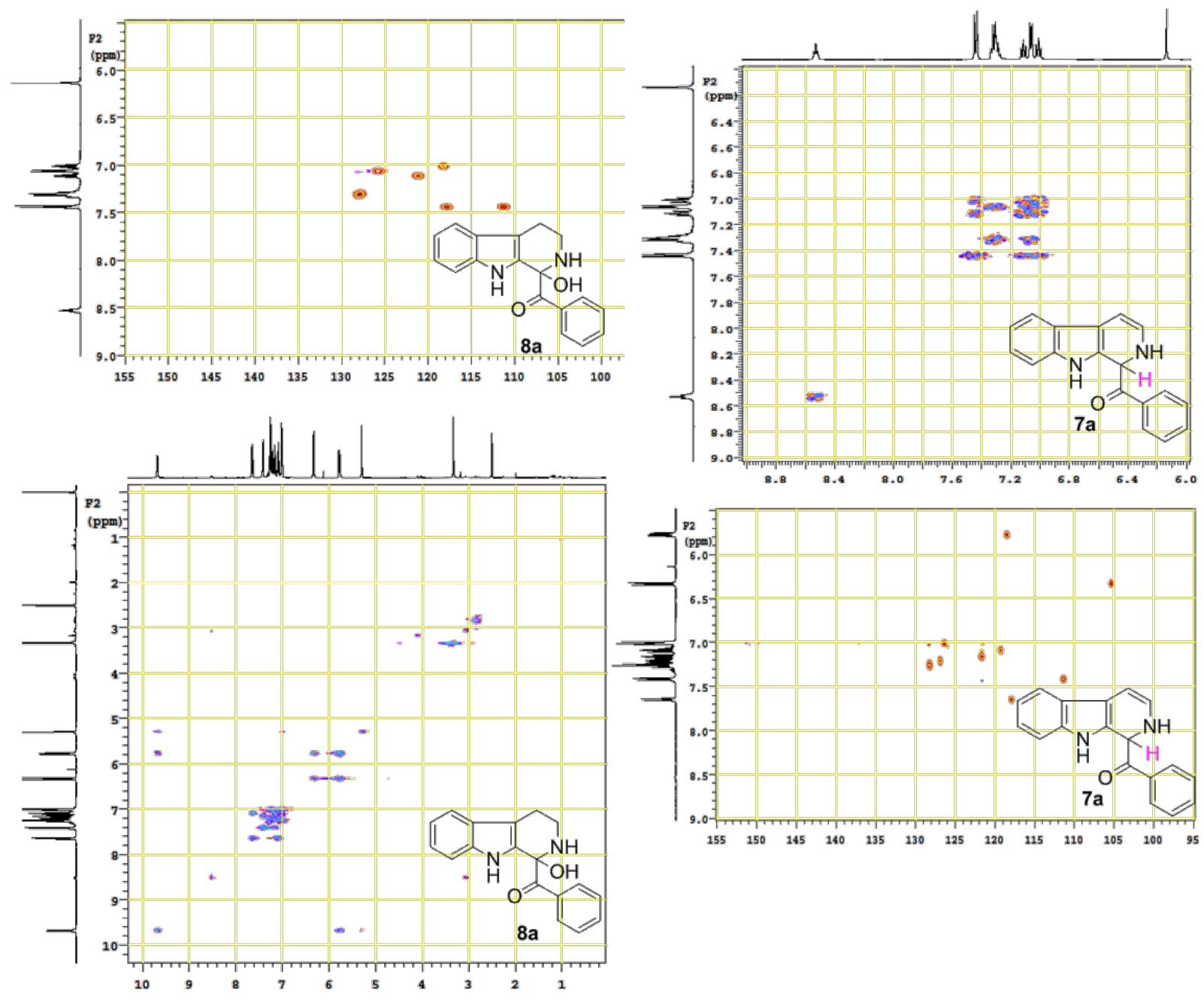

Figure 2: COSY and HSQC of $\mathbf{8 a}$ and $\mathbf{7 a}$.

Dihydro- $\beta$-carboline 7 was then subjected to an aromatization reaction to obtain eudistomin $\mathrm{Y}(\mathbf{6})$. The aromatization reaction was attempted with oxidizing agents such as DDQ and $\mathrm{MnO}_{2}$ as per the reported conditions in literature. The formation of product $\mathbf{6}$ was confirmed by LCMS analysis of the crude reaction mixture. However under these conditions product 6 was observed in less than $5 \%$ and our attempt to isolate the eudistomin Y (6) in pure form was not successful. The oxidation of compound 7 with oxygen, $\mathrm{KMnO}_{4}, \mathrm{MnO}_{2}$ and TBHP as well as dehydrogenation with DBU in different solvents at various temperatures also failed and did not yield the required product. Attempted oxidation of compound 7 under microwave irradiation conditions was also not successful. Probably under all aforementioned conditions, formation of the stable enol $\mathbf{2 1}$ might have retarded the aromatization during the course of dehydrogenation reaction of dihydro- $\beta$-carboline 7 .
Utilizing the oxidative amidation Bischler-Napieralski reaction conditions we have synthesized a number of dihydro- $\beta$-carbolines (Table 2) in moderate to good yields. The structures of these compounds were confirmed by spectral and analytical methods.

\section{Conclusion}

In conclusion, we have developed an oxidative amidation Bischler-Napieralski reaction methodology for the synthesis of dihydroeudistomin Y (7a-7i). A number of 1-benzoyl dihydro$\beta$-carboline derivates have been synthesized as a part of these studies. The oxidative amidation Bischler-Napieralski reaction provides a simple and direct method for the synthesis of carbolines, which are otherwise synthesized by multistage reactions utilizing starting materials which are not readily available. 
Table 2: Synthesis of dihydro- $\beta$-carbolines ${ }^{a}$.

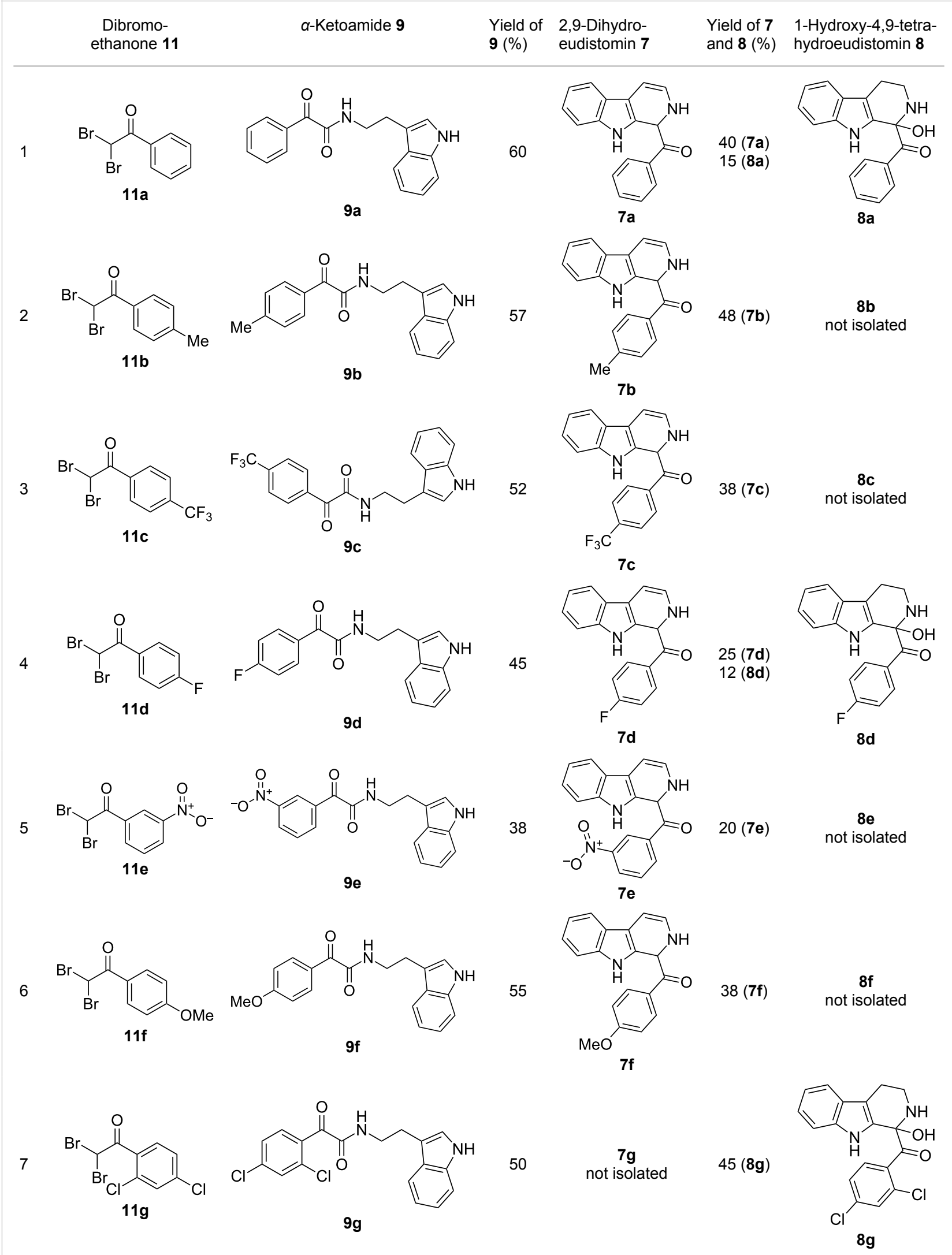


8<smiles>O=C(c1ccc(OC(Br)(Br)Br)cc1)C(Br)Br</smiles>

9<smiles>O=C(c1ccc(-c2ccccc2)cc1)C(Br)Br</smiles>

$11 \mathrm{i}$

10<smiles>O=C(c1cccs1)C(Br)Br</smiles>

11j<smiles>O=C(NCCc1c[nH]c2ccccc12)C(=O)c1ccc(OC(=O)c2ccccc2)cc1</smiles><smiles>O=C(NCCc1c[nH]c2ccccc12)C(=O)c1ccc(-c2ccccc2)cc1</smiles>

9i<smiles>O=C(NCCc1c[nH]c2ccccc12)C(=O)c1cccs1</smiles>

9j<smiles>CC(C)(C)Oc1ccc(C(=O)C2NC=Cc3c2[nH]c2ccccc32)cc1</smiles>

$8 \mathrm{~h}$ not isolated

60<smiles>O=C(c1ccc(-c2ccccc2)cc1)C1NC=Cc2c1[nH]c1ccccc21</smiles>

$35(7 i)$

$20(8 i)$

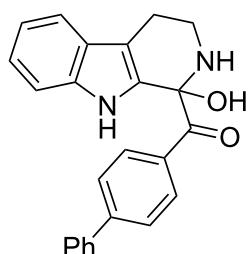

$8 \mathbf{i}$

42
$7 \mathrm{j}$ not isolated
$42(8 \mathbf{j})$

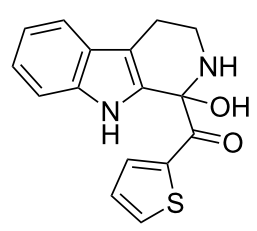

8j

${ }^{a}$ All products were characterized by ${ }^{1} \mathrm{H}$ NMR, ${ }^{13} \mathrm{C}$ NMR, Mass and IR.

\section{Experimental}

\section{General procedure for the synthesis of a-ketoamides (9a-9j)}

A mixture of 2,2-dibromo-1-phenylethanone (11a, $6.0 \mathrm{~g}$, $21.6 \mathrm{mmol})$ and sodium iodide $(6.48 \mathrm{~g}, 43.2 \mathrm{mmol})$ in dimethyl sulfoxide $(30.0 \mathrm{~mL})$ at $25-45^{\circ} \mathrm{C}$ was stirred for $40-50$ minutes. Triethylamine $(6.55 \mathrm{~g}, 64.8 \mathrm{mmol})$ and tryptamine $(\mathbf{1 0}, 3.45 \mathrm{~g}$, $21.6 \mathrm{mmol}$ ) were then added to the mixture under a $\mathrm{N}_{2}$ atmosphere and it was stirred for $1-2 \mathrm{~h}$ at $25-45^{\circ} \mathrm{C}$. Then cumene hydroperoxide ( $88 \% n$-hexane solution, $3.73 \mathrm{~g}, 21.6 \mathrm{mmol}$ ) was added to the mixture over a period of five minutes (exothermic reaction), and it was further stirred for another $3-6 \mathrm{~h}$ at the same temperature. After completion of the reaction (TLC), ice water was added to the mixture and extracted with DCM $(2 \times 50 \mathrm{~mL})$. The DCM layer was washed with aq sodium bisulfite solution $(50 \mathrm{~mL})$ followed by brine $(50 \mathrm{~mL})$ and $\mathrm{H}_{2} \mathrm{O}$ $(2 \times 60 \mathrm{~mL})$. The organic layer was dried (anhyd. $\left.\mathrm{Na}_{2} \mathrm{SO}_{4}\right)$ and evaporated to dryness under reduced pressure. The obtained crude product was subjected to CC purification and afforded the desired product in moderate to good yields.

$\boldsymbol{N}$-(2-(1H-Indol-3-yl)ethyl)-2-oxo-2-phenylacetamide (9a): $60 \%$ yield; mp $135-136.7{ }^{\circ} \mathrm{C}$; IR $\left(\mathrm{cm}^{-1}\right): 3408,3386,2927$, 2862, 1663, 1531, 1288, 1248, 1218, 1093, 1080, 1071, 838, 742; ${ }^{1} \mathrm{H}$ NMR $\left(400 \mathrm{MHz}, \mathrm{CDCl}_{3}\right) \delta_{\mathrm{H}} 8.32(\mathrm{~d}, J=8 \mathrm{~Hz}, 2 \mathrm{H})$, $8.05(\mathrm{~s}, 1 \mathrm{H}, \mathrm{NH}), 7.63(\mathrm{dd}, J=8.4,6.8 \mathrm{~Hz}, 2 \mathrm{H}), 7.47$ (t, $J=8$ $\mathrm{Hz}, 2 \mathrm{H}), 7.38$ (d, $J=8 \mathrm{~Hz}, 1 \mathrm{H}), 7.22(\mathrm{dd}, J=6.0,7.2 \mathrm{~Hz}, 1 \mathrm{H})$, $7.15(\mathrm{dd}, J=1.2,6.8 \mathrm{~Hz}, 1 \mathrm{H}), 7.12(\mathrm{dd}, J=2.0,8.8 \mathrm{~Hz}, 1 \mathrm{H})$, 7.10 (s, 1H), 3.75 (q, $J=6.8 \mathrm{~Hz}, 2 \mathrm{H}), 3.09$ (t, $J=6.8 \mathrm{~Hz}, 2 \mathrm{H})$; ${ }^{13} \mathrm{C}$ NMR (100 MHz, DMSO- $\left.d_{6}\right) \delta_{\mathrm{c}} 190.4,164.8,136.1,134.3$, 132.8, 129.6 (2C), 128.7 (2C), 127.1, 122.8, 120.8, 118.2 (2C), 111.2 (2C), 40.7, 24.7; MS m/z (\%): $293(\mathrm{M}+1), 315$ (M $+23)$.

N-(2-(1 H-Indol-3-yl)ethyl)-2-oxo-2-( $p$-tolyl)acetamide (9b): 57\% yield; mp $116.8-118.1{ }^{\circ} \mathrm{C}$; IR $\left(\mathrm{cm}^{-1}\right): 3340,3255,3093$, 2841, 1661, 1644, 1514, 1457, 1310, 1263, 1224, 1169, 1024, 849, 798, 742; ${ }^{1} \mathrm{H}$ NMR $\left(400 \mathrm{MHz}, \mathrm{CDCl}_{3}\right) \delta_{\mathrm{H}} 8.24(\mathrm{~d}, J=8$ $\mathrm{Hz}, 2 \mathrm{H}), 8.06$ (s, 1H, NH), 7.64 (d, $J=8 \mathrm{~Hz}, 1 \mathrm{H}), 7.39$ (d, $J=8$ $\mathrm{Hz}, 1 \mathrm{H}), 7.21$ (t, $J=7.2 \mathrm{~Hz}, 2 \mathrm{H}), 7.13$ (t, $J=7.2 \mathrm{~Hz}, 2 \mathrm{H}), 7.07$ $(\mathrm{d}, J=2.4 \mathrm{~Hz}, 1 \mathrm{H}), 3.73(\mathrm{~d}, J=6.4 \mathrm{~Hz}, 2 \mathrm{H}), 3.07$ (t, $J=6.8 \mathrm{~Hz}$, $2 \mathrm{H}), 2.42(\mathrm{~s}, 3 \mathrm{H}):{ }^{13} \mathrm{C}$ NMR (100 MHz, DMSO- $\left.d_{6}\right) \delta_{\mathrm{c}} 190.1$, 165.2, 145.2, 136.2, 132.3, 129.8, 129.4, 127.1, 125.7, 122.8, 120.9118 .3 (2C), 114.2, 111.3 (2C), 55.7, 24.8, 21.4; MS m/z (\%): $307.5(M+1), 329.5(M+23)$.

\section{$\mathrm{N}$-(2-(1H-Indol-3-yl)ethyl)-2-oxo-2-(4-(trifluoromethyl)phe-} nyl)acetamide (9c): $52 \%$ yield; $\mathrm{mp} 139.8-140.1^{\circ} \mathrm{C}$; IR $\left(\mathrm{cm}^{-1}\right)$ : 3338, 2938, 2842, 1640, 1334, 1224, 1157, 1135, 1075, 935, $842,794,747 ;{ }^{1} \mathrm{H}$ NMR $\left(400 \mathrm{MHz}, \mathrm{CDCl}_{3}\right) \delta_{\mathrm{H}} 8.42(\mathrm{~d}, J=8$ $\mathrm{Hz}, 2 \mathrm{H}), 8.06$ (s, 1H, NH), 7.73 (d, $J=8 \mathrm{~Hz}, 2 \mathrm{H}), 7.64(\mathrm{~d}, J=8$ $\mathrm{Hz}, 1 \mathrm{H}), 7.40(\mathrm{~d}, J=8 \mathrm{~Hz}, 1 \mathrm{H}), 7.22(\mathrm{t}, J=7.2 \mathrm{~Hz}, 1 \mathrm{H}), 7.14$ (t, $J=7.2 \mathrm{~Hz}, 2 \mathrm{H}), 3.75(\mathrm{~d}, J=6.8 \mathrm{~Hz}, 2 \mathrm{H}), 3.09(\mathrm{t}, J=6.8 \mathrm{~Hz}$, $2 \mathrm{H}) ;{ }^{13} \mathrm{C}$ NMR $\left(100 \mathrm{MHz}, \mathrm{DMSO}-d_{6}\right) \delta_{\mathrm{c}} 189.0,163.7,136.1$ 
(2C), 130.4 (2C), 127.1, 125.6 (2C), 122.8, 120.8118 .2 (2C), 111.2 (2C), 39.2, 24.7; MS m/z (\%): $383(\mathrm{M}+1), 405$ (M $+23)$.

$\mathrm{N}$-(2-(1 H-Indol-3-yl)ethyl)-2-(4-fluorophenyl)-2-oxoacetamide (9d): $45 \%$ yield; $\mathrm{mp} 138.5-141.2{ }^{\circ} \mathrm{C}$; IR $\left(\mathrm{cm}^{-1}\right)$ : 3338 , 3335, 2923, 2858, 1682, 1651, 1595, 1522, 1352, 1273, 1228 , $1152,850,798,742 ;{ }^{1} \mathrm{H}$ NMR $\left(400 \mathrm{MHz}, \mathrm{CDCl}_{3}\right) \delta_{\mathrm{H}} 8.41(\mathrm{dd}$, $J=5.2,2 \mathrm{~Hz}, 2 \mathrm{H}), 8.09$ (s, 1H, NH), 7.63 (d, $J=7.6 \mathrm{~Hz}, 1 \mathrm{H})$, $7.38(\mathrm{~d}, J=8 \mathrm{~Hz}, 1 \mathrm{H}), 7.21(\mathrm{t}, J=7.2 \mathrm{~Hz}, 1 \mathrm{H}), 7.14$ (t, $J=2$ $\mathrm{Hz}, 1 \mathrm{H}), 7.13$ to $7.11(\mathrm{~m}, 1 \mathrm{H}), 7.10(\mathrm{t}, J=2 \mathrm{~Hz}, 1 \mathrm{H}), 7.06(\mathrm{~d}, J$ $=1.6 \mathrm{~Hz}, 1 \mathrm{H}), 3.73(\mathrm{~d}, J=6.4 \mathrm{~Hz}, 2 \mathrm{H}), 3.07(\mathrm{t}, J=6.8 \mathrm{~Hz}, 2 \mathrm{H})$; ${ }^{13} \mathrm{C}$ NMR $\left(100 \mathrm{MHz}, \mathrm{CDCl}_{3}\right) \delta_{\mathrm{c}} 188.6,164.3,163.0,136.1$, $132.9,132.7,129.6,127.1,122.8,120.9,116.1,115.7,118.2$ (2C), 111.2 (2C), 39.2, 24.7; MS m/z (\%): $311.5(\mathrm{M}+1), 333.4$ $(\mathrm{M}+23)$.

$\mathrm{N}$-(2-(1 H-Indol-3-yl)ethyl)-2-(3-nitrophenyl)-2-oxoacetamide (9e): $38 \%$ yield; mp $138.1-139.5^{\circ} \mathrm{C}$; IR $\left(\mathrm{cm}^{-1}\right): 3364$, 3331, 3121, 2860, 1662, 1523, 1346, 1263, 1212, 1098, 815, 752, 725; ${ }^{1} \mathrm{H}$ NMR $\left(400 \mathrm{MHz}, \mathrm{CDCl}_{3}\right) \delta_{\mathrm{H}} 9.13(\mathrm{~s}, 1 \mathrm{H}), 8.68(\mathrm{~d}$, $J=7.2 \mathrm{~Hz}, 1 \mathrm{H}), 8.46(\mathrm{~d}, J=7.2 \mathrm{~Hz}, 2 \mathrm{H}), 8.09$ (s, 1H, NH), 7.67 $(\mathrm{dd}, J=8 \mathrm{~Hz}, 1 \mathrm{H}), 7.40(\mathrm{~d}, J=8 \mathrm{~Hz}, 1 \mathrm{H}), 7.25$ to $7.10(\mathrm{~m}, 3 \mathrm{H}$, $\operatorname{ArH}), 3.77$ (d, $J=6.6 \mathrm{~Hz}, 2 \mathrm{H}), 3.10$ (t, $J=7 \mathrm{~Hz}, 2 \mathrm{H})$; ${ }^{13} \mathrm{C}$ NMR $\left(100 \mathrm{MHz}, \mathrm{DMSO}-d_{6}\right) \delta_{\mathrm{c}} 187.3,162.8,147.7$, $136.1,135.8,134.1,130.4,128.2,127.2,124.2,122.7,120.8$, 118.2, 111.2, 39.2, 24.7; MS m/z (\%): $338.5\left(\mathrm{M}^{+}\right), 360.5\left(\mathrm{M}^{+}\right.$, 23).

\section{General procedure for the synthesis of dihy- droeudistomin $(\mathbf{7 a}-\mathbf{7 j})$}

To a stirred solution of $\mathrm{N}$-(2-(1H-indol-3-yl)ethyl)-2-oxo-2phenylacetamide (9a, $2.0 \mathrm{~g}, 6.8 \mathrm{mmol})$ in diethyl ether $(20 \mathrm{~mL})$ was added $48 \%$ boron trifluoride etherate $(10.1 \mathrm{~g}, 34.2 \mathrm{mmol})$ in a round-bottom flask under a $\mathrm{N}_{2}$ atmosphere, and the mixture was stirred at $25-30{ }^{\circ} \mathrm{C}$ for $2 \mathrm{~h}$. After TLC indication for absence of starting material toluene $(20 \mathrm{~mL})$ was added. Then mixture was heated to $55-70{ }^{\circ} \mathrm{C}$ and it was maintained under stirring at that temperature for $4-8 \mathrm{~h}$ (TLC). The mixture was cooled to $25-30{ }^{\circ} \mathrm{C}$ and it was poured into a pre-cooled sat. $\mathrm{NaHCO}_{3}$ solution $(160 \mathrm{~mL})$ at $10{ }^{\circ} \mathrm{C}$. The product was then extracted with ethyl acetate $(2 \times 25 \mathrm{~mL})$ and the organic layer was washed with $10 \% \mathrm{NaHCO}_{3}$ solution $(25 \mathrm{~mL})$ and dried (anhyd. $\mathrm{Na}_{2} \mathrm{SO}_{4}$ ). The solvent was distilled off under reduced pressure and the crude residue was subjected to column chromatography purification. The product was obtained in good to moderate yields.

(2,9-Dihydro-1H-pyrido[3,4-b]indol-1-yl)(phenyl)methanone (7a): $40 \%$ yield; mp $228.5-230.6{ }^{\circ} \mathrm{C}$; ${ }^{1} \mathrm{H}$ NMR $(400 \mathrm{MHz}$, DMSO- $\left.d_{6}\right) \delta_{\mathrm{H}} 11.37$ (br s, $\left.1 \mathrm{H}, \mathrm{NH}\right), 9.68(\mathrm{~d}, J=5.2 \mathrm{~Hz}, 1 \mathrm{H})$, $7.65(\mathrm{~d}, J=8.0 \mathrm{~Hz}, 1 \mathrm{H}), 7.42(\mathrm{~d}, J=7.6 \mathrm{~Hz}, 1 \mathrm{H}), 7.26(\mathrm{t}, J=$ $7.4 \mathrm{~Hz}, 2 \mathrm{H}), 7.22(\mathrm{~d}, J=7.2 \mathrm{~Hz}, 1 \mathrm{H}), 7.16(\mathrm{t}, J=7.6 \mathrm{~Hz}, 1 \mathrm{H})$, 7.08 (t, $J=7.2 \mathrm{~Hz}, 1 \mathrm{H}), 7.01(\mathrm{~d}, J=7.2 \mathrm{~Hz}, 2 \mathrm{H}), 6.34$ (d, $J=8.8$ $\mathrm{Hz}, 1 \mathrm{H}), 5.77(\mathrm{dd}, J=3.2 \& 5.6 \mathrm{~Hz}, 1 \mathrm{H}), 5.29(\mathrm{~s}, 1 \mathrm{H})$; ${ }^{13} \mathrm{C}$ NMR $\left(100 \mathrm{MHz}, \mathrm{DMSO}-d_{6}\right) \delta_{\mathrm{c}} 167.1,136.4,136.1,130.8$, 128.5 (2C), 127.2, 126.6 (2C), 125.9, 122.0, 119.6, 118.7, 118.2, 111.6, 109.9, 105.7, 53.2; MS $m / z(\%): 275.1(\mathrm{M}+1)$, $297.2(\mathrm{M}+23)$; HRMS (EI) $m / z$ : $[\mathrm{M}]^{+}$calcd for $\mathrm{C}_{18} \mathrm{H}_{15} \mathrm{~N}_{2} \mathrm{O}$, 275.1184; found, 275.1173 .

(1-Hydroxy-2,3,4,9-tetrahydro-1 $H$-pyrido[3,4-b]indol-1yl)(phenyl)methanone (8a): $15 \%$ yield; $\mathrm{mp} 138.5-141.2{ }^{\circ} \mathrm{C}$; ${ }^{1} \mathrm{H}$ NMR $\left(400 \mathrm{MHz}, \mathrm{DMSO}-d_{6}\right) \delta_{\mathrm{H}} 10.93(\mathrm{~s}, 1 \mathrm{H}), 8.52$ (t, $J=$ $5.2 \mathrm{~Hz}, 1 \mathrm{H}, \mathrm{NH}), 7.44(\mathrm{~d}, J=6.8 \mathrm{~Hz}, 2 \mathrm{H}), 7.33$ to $7.26(\mathrm{~m}, 3 \mathrm{H}$, ArH), $7.11(\mathrm{t}, J=6.0 \mathrm{~Hz}, 1 \mathrm{H}), 7.06(\mathrm{~d}, J=5.6 \mathrm{~Hz}, 2 \mathrm{H}), 7.00$ (t, $J=6.0 \mathrm{~Hz}, 1 \mathrm{H}), 6.13\left(\mathrm{~s}, 1 \mathrm{H}, \mathrm{D}_{2} \mathrm{O}\right.$ exchangable proton $)$, 3.10-3.02 (m, 2H), 2.88-2.49 (m, 2H); ${ }^{13} \mathrm{C}$ NMR (100 MHz, DMSO $\left.-d_{6}\right) \delta_{\mathrm{c}} 173.9,143.9,135.0,131.0,128.3(2 \mathrm{C}), 127.8$, $127.8,126.0$ (2C), 121.3, 118.5, 117.9, 111.5, 108.4, 75.6 (alkoxy carbon), 38.3, 25.2. HRMS (EI) $\mathrm{m} / \mathrm{z}$ : [M] ${ }^{+}$calcd for $\mathrm{C}_{18} \mathrm{H}_{17} \mathrm{~N}_{2} \mathrm{O}_{2}$, 293.1290; found, 293.1333; HRMS of dehydrated 8a (EI) $m / z$ : $[\mathrm{M}]^{+}$calcd for $\mathrm{C}_{18} \mathrm{H}_{15} \mathrm{~N}_{2} \mathrm{O}, 275.1184$; found, 275.1220 .

\section{(2,9-Dihydro-1H-pyrido[3,4-b]indol-1-yl)(p-tolyl)methanone} (7b): $48 \%$ yield; $\mathrm{mp} 213.2-215.7^{\circ} \mathrm{C}$; IR $\left(\mathrm{cm}^{-1}\right)$ : 3222,3179 , 3043, 2940, 1656, 1625, 1558, 1479, 1335, 1246, 1065, 802, 768, 739; ${ }^{1} \mathrm{H}$ NMR (400 MHz, DMSO- $\left.d_{6}\right) \delta_{\mathrm{H}} 11.36$ (br s, $1 \mathrm{H}$, $\mathrm{NH}), 9.64(\mathrm{~d}, J=4.8 \mathrm{~Hz}, 1 \mathrm{H}), 7.64(\mathrm{~d}, J=7.6 \mathrm{~Hz}, 1 \mathrm{H}), 7.41$ (d, $J=8.0 \mathrm{~Hz}, 1 \mathrm{H}), 7.15(\mathrm{t}, J=7.6 \mathrm{~Hz}, 1 \mathrm{H}), 7.10(\mathrm{~d}, J=7.6 \mathrm{~Hz}$, $1 \mathrm{H}), 7.06(\mathrm{~d}, J=7.6 \mathrm{~Hz}, 2 \mathrm{H}), 6.89(\mathrm{~d}, J=8.0 \mathrm{~Hz}, 2 \mathrm{H}), 6.32(\mathrm{~d}$, $J=8.4 \mathrm{~Hz}, 1 \mathrm{H}), 5.76(\mathrm{dd}, J=3.6 \& 5.6 \mathrm{~Hz}, 1 \mathrm{H}), 5.23(\mathrm{~s}, 1 \mathrm{H})$, $2.20(\mathrm{~s}, 3 \mathrm{H}) ;{ }^{13} \mathrm{C} \mathrm{NMR}\left(100 \mathrm{MHz}, \mathrm{CDCl}_{3}+\mathrm{DMSO}-d_{6}\right) \delta_{\mathrm{c}}$ $166.3,135.1,134.9,131.5,129.7,127.5(2 \mathrm{H}), 125.4(2 \mathrm{C})$, 124.6, 120.3, 118.1, 117.1, 116.5, 110.1, 108.5, 104.5, 51.6, 19.5; MS $m / z(\%): 289.5(\mathrm{M}+1), 311.5(\mathrm{M}+23)$; HRMS (EI) $\mathrm{m} / z:[\mathrm{M}]^{+}$calcd for $\mathrm{C}_{19} \mathrm{H}_{17} \mathrm{~N}_{2} \mathrm{O}, 289.1341$; found, 289.1329 .

(2,9-Dihydro-1H-pyrido[3,4-b]indol-1-yl)(4-(trifluoromethyl)phenyl)methanone (7c): $38 \%$ yield. mp $162-164{ }^{\circ} \mathrm{C}$; IR $\left(\mathrm{cm}^{-1}\right): 3222,3185,3042,2937,2835,1657,1624,1509$, 1456, 1249, 1177, 1055, 1032, 848, 810, 770, 742; ${ }^{1} \mathrm{H}$ NMR $\left(400 \mathrm{MHz}, \mathrm{CDCl}_{3}\right) \delta_{\mathrm{H}} 8.10(\mathrm{~s}, 1 \mathrm{H}), 7.69(\mathrm{~d}, J=7.6 \mathrm{~Hz}, 1 \mathrm{H})$, $7.58(\mathrm{~d}, J=8.0 \mathrm{~Hz}, 2 \mathrm{H}), 7.38(\mathrm{t}, J=8.0 \mathrm{~Hz}, 2 \mathrm{H}), 7.29-7.21(\mathrm{~m}$, $4 \mathrm{H}), 6.48(\mathrm{~d}, J=8.8 \mathrm{~Hz}, 1 \mathrm{H}), 5.94(\mathrm{dd}, J=3.6 \& 5.6 \mathrm{~Hz}, 1 \mathrm{H})$, $5.19(\mathrm{~s}, 1 \mathrm{H}) ;{ }^{13} \mathrm{C}$ NMR $\left(100 \mathrm{MHz}, \mathrm{DMSO}-d_{6}\right) \delta_{\mathrm{c}} 166.0,140.5$, $136.3,129.9,127.9,127.6,127.4$ (2C), 125.6 (doublet), 125.3 (2C), 121.9, 119.5, 118.7, 118.1, 111.6, 109.9, 105.7, 52.7; MS $\mathrm{m} / \mathrm{z}(\%): 343(\mathrm{M}+1)$; HRMS (EI) $\mathrm{m} / z:[\mathrm{M}]^{+}$calcd for $\mathrm{C}_{19} \mathrm{H}_{14} \mathrm{~N}_{2} \mathrm{OF}_{3}, 343.1058$; found, 343.1038 . 
(2,9-dihydro-1 $H$-pyrido [3,4-b]indol-1-yl)(4-fluorophenyl)methanone (7d): $25 \%$ yield; $\mathrm{mp} 230.6-232.2{ }^{\circ} \mathrm{C}$; IR $\left(\mathrm{cm}^{-1}\right)$ : 3351, 3279, 3083, 2895, 2839, 1659, 1605, 1508, 1459 , 1304, 1234, 1161, 1072, 956, 837, 747; ${ }^{1} \mathrm{H}$ NMR $(400 \mathrm{MHz}$, $\left.\mathrm{CDCl}_{3}\right) \delta_{\mathrm{H}} 8.12(\mathrm{~s}, 1 \mathrm{H}), 7.68(\mathrm{~d}, J=7.6 \mathrm{~Hz}, 1 \mathrm{H}), 7.37(\mathrm{~d}, J=$ $7.6 \mathrm{~Hz}, 1 \mathrm{H}), 7.27-7.19(\mathrm{~m}, 4 \mathrm{H}, \mathrm{ArH}), 7.00(\mathrm{t}, J=8.8 \mathrm{~Hz}, 2 \mathrm{H})$, $6.51(\mathrm{~d}, J=8.4 \mathrm{~Hz}, 1 \mathrm{H}), 5.94(\mathrm{dd}, J=3.6 \& 4.8 \mathrm{~Hz}, 1 \mathrm{H}), 5.08$ $(\mathrm{s}, 1 \mathrm{H}) ;{ }^{13} \mathrm{C}$ NMR $\left(100 \mathrm{MHz}, \mathrm{DMSO}-d_{6}\right) \delta_{\mathrm{c}} 166.7,162.4$, 136.3, 132.1, 132.1, 130.6 (doublet), 128.6 (2C), 128.6, 125.7, 121.9, 119.5, 118.7, 118.1, 115.3, 115.1, 111.6, 109.8, 105.6, 52.3; MS $m / z$ (\%): $293(\mathrm{M}+1)$; HRMS (EI) $m / z:[\mathrm{M}]^{+}$calcd for $\mathrm{C}_{18} \mathrm{H}_{14} \mathrm{~N}_{2} \mathrm{OF}$, 293.1090; found, 293.1073.

(4-Fluorophenyl)(1-hydroxy-2,3,4,9-tetrahydro-1 Hpyrido $[3,4-b]$ indol-1-yl)methanone (8d): $12 \%$ yield; $\mathrm{mp}$ $185-187^{\circ} \mathrm{C}$; IR $\left(\mathrm{cm}^{-1}\right): 3268,3178,3041,2934,1658,1626$, 1506, 1471, 1335, 1239, 1225, 1156, 1014, 976, 840, 816, 738; ${ }^{1} \mathrm{H}$ NMR $\left(400 \mathrm{MHz}, \mathrm{CDCl}_{3}+\mathrm{DMSO}-d_{6}\right) \delta_{\mathrm{H}} 9.65(\mathrm{~s}, 1 \mathrm{H}), 7.89$ (t, $J=6.8 \mathrm{~Hz}, 1 \mathrm{H}), 7.47(\mathrm{t}, J=9.4 \mathrm{~Hz}, 3 \mathrm{H}), 7.22-7.08(\mathrm{~m}, \mathrm{H}$, $\mathrm{ArH}), 6.96(\mathrm{t}, J=8.8 \mathrm{~Hz}, 2 \mathrm{H}), 5.92(\mathrm{~s}, 1 \mathrm{H}), 3.24-3.19(\mathrm{~m}, 2 \mathrm{H})$, $2.94(\mathrm{~m}, 2 \mathrm{H}) ;{ }^{13} \mathrm{C}$ NMR $\left(100 \mathrm{MHz}, \mathrm{DMSO}-d_{6}\right) \delta_{\mathrm{c}} 173.6,140.3$, 135.0, 130.8, 128.2, 128.1, 127.7, 121.4, 118.5, 118.0, 115.3, 114.9, 111.5, 108.5, 75.0, 25.1; MS m/z (\%): $293(\mathrm{M}+1), 315$ $(\mathrm{M}+23)$; HRMS (EI) $m / z:[\mathrm{M}]^{+}$calcd for $\mathrm{C}_{18} \mathrm{H}_{14} \mathrm{~N}_{2} \mathrm{OF}$, 293.1090; found, 293.1070 .

\section{Supporting Information}

\section{Supporting Information File 1 \\ Experimental and analytical data. \\ [http://www.beilstein-journals.org/bjoc/content/ \\ supplementary/1860-5397-10-45-S1.pdf]}

\section{Acknowledgements}

The authors would like to thank Dr. Vilas H. Dahanukar, Dr.Reddy's Laboratories for useful discussions and constant encouragement. We also thank Gajanan S. Botre from the analytical department, Dr. Reddy's Laboratories, for providing the HRMS data.

\section{References}

1. Cao, R.; Peng, W.; Wang, Z.; Xu, A. Curr. Med. Chem. 2007, 14, 479-500. doi:10.2174/092986707779940998

2. Rinehart, K. L., Jr.; Kobayashi, J.; Harbour, G. C.; Hughes, R. G., Jr.; Mizsak, S. A.; Scahill, T. A. J. Am. Chem. Soc. 1984, 106, 1524-1526. doi:10.1021/ja00317a079

3. Rinehart, K. L., Jr.; Kobayashi, J.; Harbour, G. C.; Gilmore, J.; Mascal, M.; Holt, T. G.; Shield, L. S.; Lafargue, F. J. Am. Chem. Soc. 1987, 109, 3378-3387. doi:10.1021/ja00245a031
4. Rocca, P.; Marsais, F.; Godard, A.; Quéguiner, G.; Adams, L.; Alo, B. Synth. Commun. 1995, 25, 3373-3379. doi:10.1080/00397919508013858

5. Molina, P.; Fresneda, P. M.; Garcia-Zafra, S. Tetrahedron Lett. 1996, 37, 9353-9356. doi:10.1016/S0040-4039(97)82962-0

6. Kinzer, F. K.; Cardellina, J. H., II. Tetrahedron Lett. 1987, 28, 925-926. doi:10.1016/S0040-4039(00)95875-1

7. Mahale, S.; Aubry, C.; Wilson, A. J.; Jenkins, P. R.; Maréchal, J.-D.; Sutcliffe, M. J.; Chaudhuri, B. Bioorg. Med. Chem. Lett. 2006, 16, 4272-4278. doi:10.1016/j.bmcl.2006.05.065

8. Callaway, J. C. J. Psychoact. Drugs 2005, 37, 151-155. doi:10.1080/02791072.2005.10399796

9. Song, Y.; Wang, J.; Teng, S. F.; Kesuma, D.; Deng, Y.; Duan, J.; Wang, J. H.; Qi, R. J.; Sim, M. M. Bioorg. Med. Chem. Lett. 2002, 12, 1129-1132. doi:10.1016/S0960-894X(02)00094-X

10. Schott, Y.; Decker, M.; Rommelspacher, H.; Lehmann, J. Bioorg. Med. Chem. Lett. 2006, 16, 5840-5843. doi:10.1016/j.bmcl.2006.08.067

11. Chen, Z.; Hu, G.; Li, D.; Chen, J.; Li, Y.; Zhou, H.; Xie, Y. Bioorg. Med. Chem. 2009, 17, 2351-2359. doi:10.1016/j.bmc.2009.02.015

12. Wang, W.; Nam, S.-J.; Lee, B.-C.; Kang, H. J. Nat. Prod. 2008, 71, 163-166. doi:10.1021/np070064o

13. Panarese, J. D.; Waters, S. P. Org. Biomol. Chem. 2013, 11, 3428-3431. doi:10.1039/c3ob40661j

14. Trieu, T. H.; Dong, J.; Zhang, Q.; Zheng, B.; Meng, T.-Z.; Lu, X.; Shi, X.-X. Eur. J. Org. Chem. 2013, 3271-3277. doi:10.1002/ejoc.201300080

15. García, M. D.; Wilson, J. A.; Emmerson, D. P. G.; Jenkins, P. R. Chem. Commun. 2006, 2586-2588. doi:10.1039/B604922B

16. García, M. D.; Wilson, J. A.; Emmerson, D. P. G.; Jenkins, P. R.; Mahale, S.; Chaudhuri, B. Org. Biomol. Chem. 2006, 4, 4478-4484. doi:10.1039/B613861F

17. Kennedy, J. P.; Breininger, M. L.; Lindsley, C. W. Tetrahedron Lett. 2009, 50, 7067-7069. doi:10.1016/j.tetlet.2009.09.180

18. Jin, H.; Zhang, P.; Bijian, K.; Ren, S.; Wan, S.; Alaoui-Jamali, M. A.; Jiang, T. Mar. Drugs 2013, 11, 1427-1439. doi:10.3390/md11051427

19. Meruva, S. B.; Raghunadh, A.; Kumar, N. A.; Vasudev, R.; Kumar, U. K. S.; Dev, R. V.; Dubey, P. K. J. Heterocycl. Chem. 2011, 48, 540-548. doi:10.1002/jhet.590

20. Wagh, M. B.; Shankar, R.; Kumar, U. K. S. Synlett 2011, 84-88. doi:10.1055/s-0030-1259098

21. Shankar, R.; Wagh, M. B.; Madhubabu, M. V.; Vembu, N.; Kumar, U. K. S. Synlett 2011, 844-848. doi:10.1055/s-0030-1259921

22. Wagh, M. B.; Shankar, R.; Mini, K.; Krishnamohan, T.; Madhubabu, M. V.; Pal, K. A.; Jayaprakash, S.; Krishna, T.; Dahanukar, V.; Kumar, U. K. S.; Gill, C. H. J. Heterocycl. Chem. 2013, 50, 49-55. doi:10.1002/jhet.991

23. Raghunadh, A.; Meruva, S. B.; Kumar, N. K.; Kumar, G. S.; Rao, L. V.; Kumar, U. K. S. Synthesis 2012, 283-289. doi:10.1055/s-0031-1289647

24. Shanmugapriya, D.; Shankar, R.; Satyanarayana, G.; Dahanukar, V. H.; Kumar, U. K. S.; Vembu, N. Synlett 2008, 2945-2950. doi:10.1055/s-0028-1087353

25. Klingenberg, J. Org. Synth., Coll. Vol. IV 1963, 110.

26. Bigelow, L. A.; Hanslick, R. S. Org. Synth., Coll. Vol. I/ 1943, 244.

27. Shankar, R.; More, S. S.; Madhubabu, M. V.; Vembu, N.; Kumar, U. K. S. Synlett 2012, 1013-1020. doi:10.1055/s-0031-1290655

28. The authors are thankful to referees for the useful suggestions. 


\section{License and Terms}

This is an Open Access article under the terms of the Creative Commons Attribution License

(http://creativecommons.org/licenses/by/2.0), which permits unrestricted use, distribution, and reproduction in any medium, provided the original work is properly cited.

The license is subject to the Beilstein Journal of Organic Chemistry terms and conditions:

(http://www.beilstein-journals.org/bjoc)

The definitive version of this article is the electronic one which can be found at:

doi:10.3762/bjoc. 10.45 